\title{
Mise en évidence des différences de croissance entre indivi- dus sauvages et introduits chez la truite Salmo trutta fario
}

\author{
J. Moreau1 \\ N. Abad'
}

Mots clés : S. trutta fario, écologie, eaux courantes, croissance

A la faveur d'une étude des populations de truites (S. trutta fario) dans le Sud du Massif Central (France), des différences de croissance entre sujets sauvages et poissons introduits de pisciculture ont été mises en évidence par la méthode de Kirmura (1980). La croissance est plus rapide chez les poissons de pisciculture. L'influence possible du comportement alimentaire est discutée.

Differences in growth pattern between indigeneous and introduced populations of Salmo trutta fario.

Keywords: S. trutta fario, ecology, rivers, growth.

Within a study on S. trutta fario populations in the south of the Massif Central (France) it was possible to indicate differences in growth pattern between local populations and stocked fishes by using the method of Kimura (1980). Growth is faster for introduced fishes. The influence of a possible difference in feeding behaviour is discussed.

\section{Introduction}

Face à l'augmentation de l'effort de pêche récréative et sportive dans plusieurs pays (Grover 1980), les empoissonnements réguliers en truites $S$. trutta fario issues de piscultures se sont généralisés. Toutefois, on peut s'interroger sur l'efficacité de ces alevinages, traduite par l'adaptation des poissons introduits, et sur l'impact qu'ils peuvent avoir sur les populations " sauvages " (Egglishaw \& Shackley 1982 ; Thibault 1983 ; FAO/EIFAC/CECPI/1983).

Une étude des populations de truites dans des affluents du Tarn (Abad 1982) a permis une approche de ce problème; le présent travail rend compte des observations destinées à étudier les différences de croissance entre poissons introduits et sauvages dans 2 rivières pour lesquelles on disposait de données sur la croissance pour plusieurs années.

1. Laboratoire d'Ichtyologie appliquee. E.N.S. Agronomique, 145 Avenue de Muret, 31076 Toulouse Cédex, France.

\section{Le milieu aquatique étudié}

Les deux rivières choisies se trouvent à l'Est du département du Tarn (France) dans la région des monts de Lacaune (fig. 1).

\subsection{Caractéristiques générales (Tableau I).}

- Le Viau prend sa source à $890 \mathrm{~m}$ d'altitude et, après un parcours de $15 \mathrm{~km}$, il se jette dans le barrage du Lauzas à 771 mètres d'altitude. Le secteur d'étude se trouve au milieu de prairies de fauche, la végétation des berges est composée de quelques arbres clairsemés, l'ensoleillement de ce secteur est important, l'eau y est incolore.

- La Vebre prend sa source à $910 \mathrm{~m}$ d'altitude. Après un parcours de $18 \mathrm{~km}$, elle se jette dans le lac artificiel du Lauzas.

Sur le secteur d'étude, la rivière coule au milieu de prairies de fauche, la végétation des berges est composée de buissons et d'arbres assez espacés, l'ensoleillement du secteur est moyen, l'eau y est incolore, le fond n'est pas colmaté et les zones de frayères y sont asséz abondantes. 
Tableau I. Caractéristiques générales des secteurs d'étude

\begin{tabular}{|c|c|c|c|}
\hline \multicolumn{2}{|l|}{ Caractéristiques } & Viau & Vèbre \\
\hline Altitude (m) & & 796 & 817 \\
\hline Largeur (m) & & 8,2 & 5,5 \\
\hline Longueur $(\mathrm{m})$ & & 500 & 325 \\
\hline Surface $\left(\mathrm{m}^{2}\right)$ & & 3540 & 1650 \\
\hline Pente mayenne $(\%)$ & & 7,8 & 7.9 \\
\hline Profondeur moyenne $(\mathrm{m})$ & & 0,65 & 0,5 \\
\hline $\begin{array}{l}\text { Granulométrie : } \\
\text { Rochers }(\%) \\
\text { Blocs }(\%) \\
\text { Galets }(\%) \\
\text { Gravet tes }(\%) \\
\text { Sable }(\%) \\
\text { Sablon }(\%) \\
\text { Limon et \& vase }(\%)\end{array}$ & $\begin{array}{rcc} & 500 & \mathrm{~mm} \\
200 \text { à } & 500 & \mathrm{~mm} \\
20 \text { à } & 200 & \mathrm{~mm} \\
2 \text { à } & 20 & \mathrm{~mm} \\
0,2 \text { à } & 2 & \mathrm{~mm} \\
0,02 \text { à } & 0,2 & \mathrm{~mm} \\
& 0,02 & \mathrm{~mm}\end{array}$ & $\begin{array}{r}10 \\
30 \\
35 \\
15 \\
5 \\
5\end{array}$ & $\begin{array}{r}5 \\
10 \\
30 \\
35 \\
10 \\
5 \\
5\end{array}$ \\
\hline $\begin{array}{l}\text { Importance de la couverture végétale } \\
\text { en \% de la surface du fond }\end{array}$ & & 75 & 40 \\
\hline Débit $\left(\mathrm{m}^{3 /} / \mathrm{s}\right)$ & & $\begin{array}{c}3.95 \\
(0,37 \text { a } 18,7)\end{array}$ & $\begin{array}{c}1,8 \\
(0,25 \text { à } 12,6)\end{array}$ \\
\hline
\end{tabular}

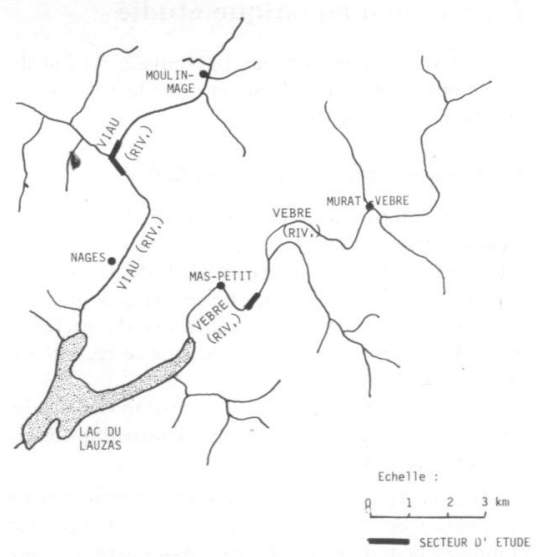

Fig. 1. Carte de situation des deux secteurs d'étude dans le bassin de l'Agout.

\subsection{Les paramètres du milieu}

Les facteurs physico-chimiques ont été étudiés par Abad (1982) ; le fait important à noter est que le pH basique $(7,2$ à 8,3$)$ et la teneur en calcium élevés $(7$ à $32 \mathrm{mg} / \mathrm{l}$ ) confèrent aux eaux des potentialités productrices moyennes ou bonnes selon la classification de Nisbet \& Verneaux (1970).

Au sujet des populations d'invertébrés aquatiques, la densité numérique est minimale au mois de septembre dans les 2 rivières ( 3500 individus $/ \mathrm{m}^{2}$ ). L'augmentation s'amorce dès le mois de novembre et le maximum est at teint en mars (8000 à 12000 individus $/ \mathrm{m}^{2}$ ). Les variations de biomasse suivent globalement celle des densités (minimum : $2 \mathrm{~g} / \mathrm{m}^{2}$ en septembre : maximum : 5 à $6 \mathrm{~g} / \mathrm{m}^{3} \mathrm{en} \mathrm{mars} \mathrm{(Abad} \mathrm{1982).}$ Il arrive que les pertes en nombre soient compensées par un gain de poids des individus, surtout au début du printemps.

En densité, le groupe éphéméroptères-diptères représente $61 \%$ : les éphéméroptères constituent 35 et $37 \%$ de l'ensemble et les diptères 27 et $23 \%$. Le 
groupe plécoptères-trichoptères constitue $23 \%$ dans le Viau et $27 \%$ du total dans la Vèbre. Les divers (coléoptères, crustacés, mollusques, plathelminthes, annélides, arachnides, odonates) représentent 12,2 $\%$ sur le Viau et $6 \%$ sur la Vèbre. Dans les deux rivières, l'ensemble de la biomasse est constituée par le groupe éphémoptères-trichoptères ; $75 \%$ sur le Viau et $76 \%$ sur la Vèbre.

\section{Matériel et Méthodes}

\subsection{Capture des poissons}

Sur chaque rivière, la zone étudiée a été choisie pour son accessibilité et la facilité d'y pratiquer la pêche électrique.

Les captures ont été pratiquées par pêche électrique selon les techniques habituelles (Vibert 1968, Lamarque \& al. 1975) aux dates suivantes: juillet 79 , février et juillet 80 , février et juillet 81 .

Le courant continu fourni par un générateur Electro-Pullman (600 V.-1, 5A) a été établi entre une électrode fixe (cathode) constituée d'un grillage de surface variable et une èlectrode mobile (anode) formée d'un anneau de cuivre de $35 \mathrm{~cm}$ de diamètre au voisinage de laquelle les poissons sont immobilisés et capturés à l'épuisette.

La technique de maniement de l'anode utilisée ici peut être qualifiée "d'active", c'est-à-dire que l'on procède par " à coups ": l'anode est rapidement portée hors de l'eau, vers l'amont, immergée quelques secondes et ramenée dans l'eau, vers les épuisettes.

Sur ces rivières de 4 à 10 mètres de largeur, les pêches ont été effectuées avec deux anodes maniées de front. L'équipe de travail se composait alors de 8 personnes.

Lors de chaque pêche, après anesthésie à l'éther monophénilique de l'éthylèneglycol, tous les individus ont été marqués de la façon suivante :

- individus de moins de $10 \mathrm{~cm}$ : ablation de la nageoire adipeuse ;

- les individus plus grands : marque métallique (Presadom plate) numérotée adaptée à leur taille et fixée à la mâchoire inférieure. Les numéros des marques utilisées ont été les suivantes : $n^{\circ} 1$ pour des poissons de 10 à $15 \mathrm{~cm}, \mathrm{n}^{\circ} 2$ pour des poissons de 15 à $25 \mathrm{~cm}$ et $n^{\circ} 3$ pour des poissons de plus de 25 $\mathrm{cm}$. Les marques $\mathrm{n}^{\circ} 0$ utilisées au début chez les poissons de moins de $8 \mathrm{~cm}$ ont dû être abandonnées car elles endommageaient la mâchoire. D'une façon générale, le marquage a été réalisé de façon à gêner au minimum la prise de nourriture par les poissons marqués comme cela est instamment suggéré par Jones (1979). Après complète récuperation de leur faculté de nage dans un bac de stabulation, les poissons ont été remis à l'eau et un opérateur a vérifié plusieurs fois, durant la journée, qu'aucun d'entre eux n'avait été entraîné par le courant et plaqué contre un filet à petites mailles placé à la limite aval du secteur d'étude. Ces filets n'ont été retirés qu'en fin de journée.

Ce protocole était destiné à éviter les dévalaisons " passives "immédiates dues au stress lié aux manipulations et à permettre aux poissons de se répartir à nouveau dans leur habitat d'originel.

\subsection{Etude de la crolssance}

La longueur totale a été mesurée à $0,1 \mathrm{~cm}$ près, et les poissons; ont été pesés à $0,5 \mathrm{~g}$ près. La déter. mination de l'âge a été basée sur la méthode scalimétrique. La méthode de prélèvements et de préparation des écailles a été celle décrite par exemple par Beall (1979). La lecture a été faite au microscope à écran.

L'étude scalimétrique a ainsi été effectuée à partir d'écailles prélevées sur des individus capturés lors de chacune des pêches évoquées plus haut. Sachant que l'éclosion survient en général en février, on a ainsi obtenu en juillet 1979, 1980 et 1981 les tailles moyennes pour les âges suivants : 6 mois, 1 an et 6 mois, 2 ans et 6 mois, etc...

En février 80 et 81 , on a, de même, obtenu les longueurs moyennes pour les âges suivants : 1 an, 2 ans, 3 ans, etc.. Au total, les lectures d'écailles ont porté sur 343 individus de toutes tailles dans le Viau et 396 dans la Vèbre. De telles observations permettent d'établir une loi de croissance en longueur tenant compte, dans une certaine mesure, des variations interannuelles de croissance souvent observées (Beall 1979).

La croissance en longueur a été exprimée par la relation de Von Bertalanffy: $L_{t}=L^{x}\left(l-e^{-k\left(t-t_{0}\right)}\right)$ qui donne la longueur du corps $\mathrm{L}_{t}$ en fonction de l'âge t. La signification des paramètres est celle habituellement admise par les auteurs (Daget \& Le Guen 1975: Moreau 1987). 
Ces paramètres ont été calculés selon la méthode de Kimura (1980) dérivée de celle d'Abramson et Tomlinson, (in Daget \& Le Guen 1975) et qui permet, par la méthode du maximum de vraisemblance, une évaluation conjointe des paramètres $L z k$ et $t_{0}$ et de leur intervalle de confiance. De plus, il est possible de tracer la région de confiance jointe de $\mathbf{L} \sim$ et $\mathrm{k}$ pour la valeur optimale de $t_{0}$. Ainsi, pour des valeurs de $t_{0}$ identiques ou au moins très proches, il est possible de comparer plusieurs courbes de croissance et de dire si elles sont significativement diférentes en vérifiant dans quelle mesure les régions de confiance se recouvrent.

Les relations âge-longueur ont été établies à partir du calcul des tailles moyennes par classe d'âge : 15 classes d'âge chez les sujets sauvages et 9 chez les poissons issus de pisciculture. En outre, pour chaque âge, la mise en ouvre de la méthode de Kimura nécessite le nombre de poissons étudiés et l'écart-type de la distribution des longueurs observées à chacun de ces âges. Un programme, disponible sur demande auprès des auteurs, a été mis au point en BASIC pour un équipement HP 9845 B disposant d'un traceur de courbes.

Les relations poids-longueur $\left(\mathbf{W} x=\mathbf{a} \mathrm{L}^{\mathrm{b}}\right)$ ont été établies à partir des mesures et des pesées individuelles et les relations âge-poids ont été déduites des deux relations précédentes. L'expression générale est :

$\mathrm{W}_{\mathrm{t}}=\mathrm{W} \times\left(1-\mathrm{e}^{-\mathrm{k}\left(\mathrm{t}-\mathrm{t}_{\mathrm{o}}\right) \mathrm{b}} \mathrm{avec}=\mathrm{aL}\right.$.

\subsection{Les individus introduits}

Il est possible de distinguer selon les critères morphologiques les individus issus de pisciculture et ceux nés en milieu sauvage. En effet, selon Abad (1982) :

“ Les sujets de pisciculture sont essentiellement repérés en raison de la coloration de leur " robe ". Il ne faut pas chercher de différences dans la ponctuation ou dans les couleurs vives de la " robe "qui peuvent varier à l'infini. La différence la plus nette se situe dans la "couleur de fond " de la "robe " que l'on met en évidence en exposant les sujets en pleine lumière dans un bac au milieu de la rivière. Les teintes vives ou sombres de la " robe ", dues au mimétisme du poisson en fonction de l'environnement dans lequel il vivait, s'estompent et laissent apparaître la couleur de "fond de robe " qui est la suivante selon l'origine du sujet :

. pisciculture : le ventre et les flancs sont d'un gris plutôt terne, le dos est gris sombre, chez les individus d'un été et gris verdâtre parsemé de "virgules * argentées chez les sujets plus àgés.

. sauvage : le ventre et les flancs vont du beige au jaune-verdattre assez lumineux, le dos est brunverdâtre ".

Ces méthodes de distinction des poissons introduits sont bien connues des gardes de pêche qui ont prêté leur concours au présent travail.

Une autre différence est la visibilité des écailles. Chez un sujet sauvage observé à une distance d'environ $50 \mathrm{~cm}$, les écailles ne se distinguent pas les unes des autres ; elles paraissent fondues les unes dans les autres et forment un revêtement uniforme. Chez un sujet de pisciculture, observé à cette même distance de $50 \mathrm{~cm}$ ou à une distance supérieure, les écailles sont nettement individualisées et bien visibles. En outre, observées au microscope, les écailles de sujets de pisciculture, en partie recouvertes d'une pellicule argentée, se révèlent plus épaisses et plus opaques que les écailles de sujets sauvages.

Les sujets de repeuplement ont donc pu être séparés de la population sauvage et chaque population a fait l'objet d'une étude distincte de la croissance linéaire et pondérale. En effet, depuis 1975, la Vèbre et le Viau reçoivent tous les ans, respectivement 7.000 et 8.000 alevins en avril et mai. Ces poissons, provenant d'une pisciculture gérée par la Fédération Départementale de Pêche du Tarn, mesurent en moyenne 4 à $5 \mathrm{~cm}$. Les individus sauvages de même âge ( 3 mois) sont, en moyenne, longs de 2 à $3 \mathrm{~cm}$, comme on peut le constater par des sondages à la pêche à l'électricité au mois de mai avant les empoissonnements évoqués ci-dessus.

Les sujets de pisciculture sont retrouvés sur le Viau jusqu'à l'âge de 5 ans et demi et sur la Vèbre jusqu'à 4 ans et demi. Ils occupent donc une place durable au sein de la population et peuvent être considérés comme adaptés ; un équilibre a pu s'établir entre la population sauvage et celle résultant des déversements successifs d'alevins de pisciculture.

\section{Résultats}

Les clefs âge-longueur obtenues sont résumées sur les figures 2 et 3 . Les relations poids-longueur 


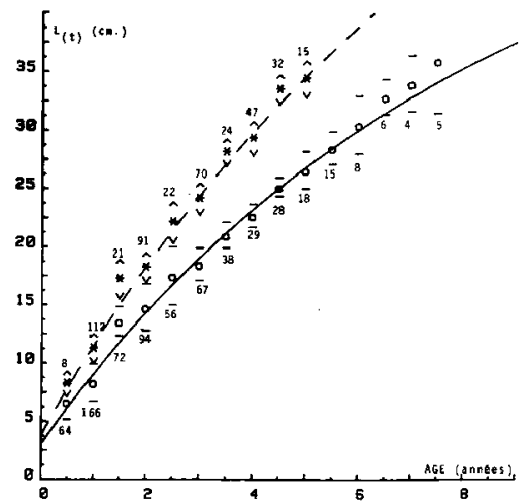

Fig. 2 : Comparaison des croissances linéaires des truites sauvages et in troduites sur le Viau.

Poissons sauvages: trait continu, o et nombre de poissons par âge mentionné sous la courbe.

Poissons introduits : trait discontinu, * et nombre de poissons par àge mentionné au-dessus de la courbe.

Les signes - et indiquent l'intervalle de confiance de la distribution des tailles.

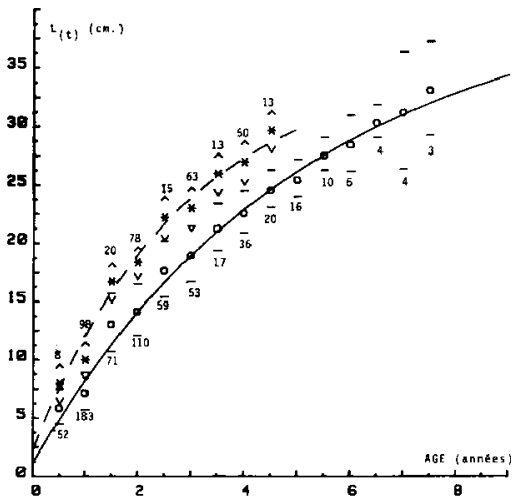

Fig. 3 : Comparaison des croissances linéatres des truites sauvages et introduites sur la Vèbre.

Méthodes de représentation comme sur la figure 2 .

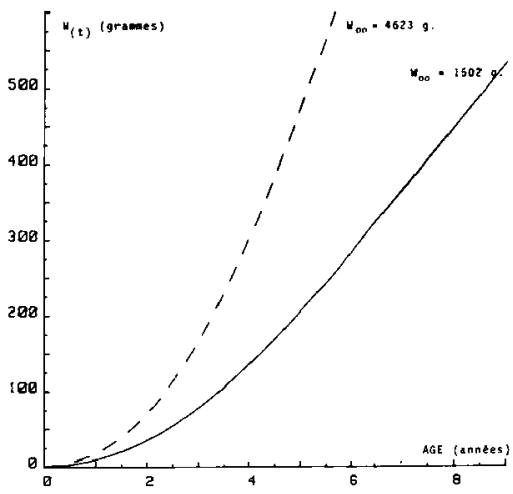

Fig. 4 : Croissance pondérale des deux populations de truites sur le Viau

Truites sauvages: trait plein

Truites introduites: trait discontinu.

destinées à l'étude de la croissance pondérale sont données ci-dessous.

\subsection{Relations poids-longueur}

* Truites sauvages :

$$
W=0,019 \mathrm{~L}^{2,826}(\mathrm{r}-0,995)
$$

* Truites issues de pisciculture

$$
W=0,012 L^{2,985}(\mathbf{r}=0,999)
$$

Vèbre

* Truites sauvages :

$$
\mathrm{W}=0,020 \mathrm{~L}^{2,793}(\mathrm{r}=0,996)
$$

* Truites issues de pisciculture

$$
W=0,015 L^{2,914}(r=0,999)
$$

\subsection{Croissances linéaires et pondérales}

Le Tableau II résume les paramètres de croissance calculés pour les diverses populations. Les croissances ainsi observées sont représentées sur les Figures 2 à 5 .

Les domaines de confiance des estimations de $\mathrm{L}$ et $\mathrm{K}$ pour les 4 populations sont tracés sur les figures 6 et 7 . 
Tableau II : Paramètres de croissance des populations étudiées (Equation de Von Bertalanffy) évaluations données avec les intervalles de confiance

\begin{tabular}{llll}
\hline & $\mathrm{L}^{*}(\mathrm{~cm})$ & $\mathrm{k}$ (annuel) & to (année) \\
\hline $\begin{array}{l}\text { T. sauvages } \\
\text { VIAU }\end{array}$ & $54.1(37.3-119.8)$ & $0,125(0.05-0.21)$ & $-0.462+0.0125$ \\
T. pisciculture & $74.3(48.4-232.8)$ & $\mathbf{0 . 1 1 5 ( 0 . 0 3 - 0 . 2 1 )}$ & $0.467+0.014$ \\
\hline $\begin{array}{l}\text { T. sauvages } \\
\text { VEBRE }\end{array}$ & $41.5(30.6-72.1)$ & $0,795(0.094-0.311)$ & $-0.137+0.009$ \\
T. pisciculture & $35.6(25.7-72.6)$ & $0.353(0.129-0.654)$ & $-0.187+0.012$ \\
\hline
\end{tabular}

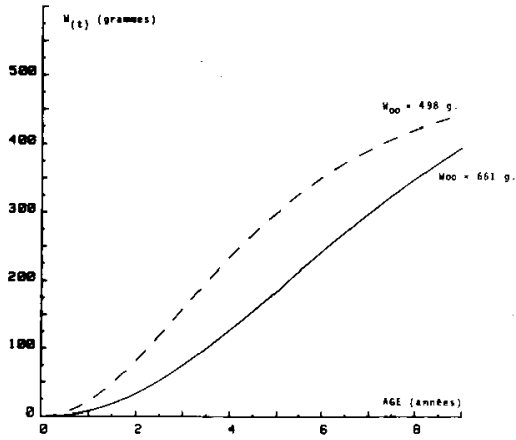

Fig. 5 : Croissance pondérale des deux populations de truites sur la Vebre.

Truites sauvages: trait plein.

Truites introduites : trait discontinu.

Ces observations révèlent que les truites issues de pisciculture et introduites au stade alevin présentent une croissance linéaire et pondérale supérieure à celle des truites sauvages, et que cette différence peut être visualisée de façon satisfaisante par la méthode de Kimura (1980).

\subsection{Vérification des résultats}

L'erreur la plus fréquente dans la lecture d'écailles de salmonidés est l'oubli du premier annulus. Par examen des écailles d'individus de l'année, il a pu être observé que celui-ci était formé en moyenne à sept circuli du centre. Une seconde erreur pourrait

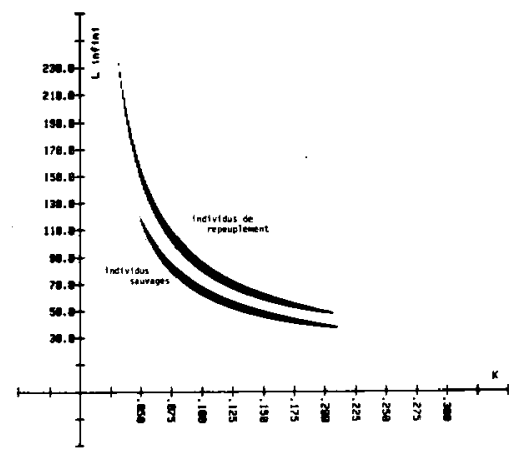

Fig. 6: Régions de confiance des évaluations de $\mathrm{K}$ et $\mathrm{L}$ par la méthode de Kimura (1980) autour des évaluations de $\mathrm{K}$ et $\mathrm{L}$ par la méthode des moindres carrés truites sauvages et introduites dans le Viau.

être due à la présence sur l'écaille de faux annuli résultant d'un stress survenant pendant la période habituelle de croissance. Ils sont assez facilement repérés, car ils ne se rencontrent généralement que sur une partie de l'écaille et/ou à une place variable sur différentes écailles d'un même poisson.

Le calcul des équations de Von Bertalanffy ayant été basé sur l'étude scalimétrique, la validité peut être vérifiée de la façon suivante. Grâce aux marquages individuels, on connaît les tailles de truites correspondant à des âges régulièrement espacés d'une année. L'intervalle de temps entre deux âges successifs étant pris comme unité, on a : 


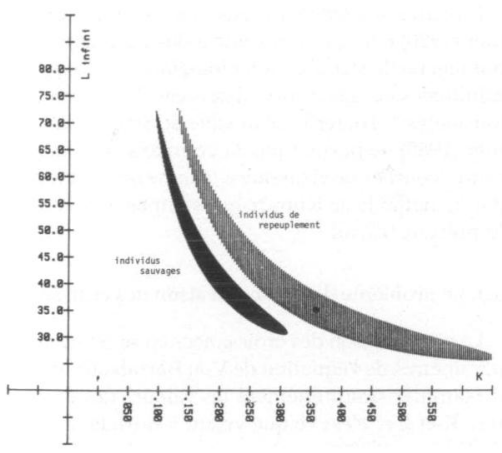

Fig. 7 : Régions de confiance des évaluations de $\mathrm{K}$ et $\mathrm{L}$ obtenues par la méthode de Kimura (1980) autour des évaluations de $\mathrm{K}$ et $\mathrm{L}$ par la méthode des moindres carres. Truites sauvages et introduites dans la Vèbre.

$\left.\mathrm{L}_{\mathrm{t}+1}=\mathrm{L}_{x}\left[1-\mathrm{e}^{-\mathrm{k}\left(1+1-\mathrm{t}_{0}\right)}\right)\right]=\mathrm{L}_{\infty}\left[1-\mathrm{e}^{-\mathrm{k}_{\mathrm{e}}-\mathrm{k}\left(\mathrm{t}-\mathrm{t}_{\mathrm{o}}\right)}\right]$

et comme : $-L_{\infty}\left[e^{\left.-k\left(t-t_{o}\right)\right]}=L_{t}-L_{\infty}\right.$

on a: $\mathbf{L}_{\mathbf{t}+\mathbf{1}}=\mathrm{L}_{\boldsymbol{n}}+\mathrm{e}^{-\mathrm{k}}\left(\mathbf{L}_{\mathrm{r}} \cdot \mathrm{L}_{\infty}\right)$

ou: $\mathbf{L}_{\mathrm{t}+1}=\mathrm{e}^{-\mathbf{k} \mathrm{t}}+\left(\mathbf{l}-\mathrm{e}^{-k}\right) \mathbf{L}_{\infty}$

Si l'on porte sur un graphique $\mathrm{L}_{t}$ en abscisse et $L_{t+1}$ en ordonée, tous les points de coordonnées $L_{t}$ et $\mathbf{L}_{t+1}$ sont situés sur une droite de pente $e^{-k}$ et coupant la première bissectrice au point, pour lequel $\mathrm{L}_{\mathrm{t}+1}=\mathrm{L}_{\mathrm{t}}=\mathrm{L}_{\infty}$

En pratique, les points observés ne sont pas rigoureusement alignés. Il faut donc calculer l'équation de la droite d'ajustement afin de déduire $\mathbf{l}$ et $\mathbf{k}$.

A titre d'exemple, pour les truites sauvages de la Vèbre (fig. 8), 287 poissons de toutes tailles parmi ceux marqués un an avant ont permis ces calculs. L'équation de cette droite, calculée par la méthode des moindres carrées est :

$\mathrm{L}_{\mathrm{t}+1}=0,850 \mathbf{L}_{\mathrm{t}}+6,679(\mathrm{r}=0,999)$

où : $L_{\infty}=\frac{6,679}{1-0,850}=44,588$

et $\mathrm{k}=-\log 0,850=0,162$

L'équation de Von Bertalanffy, basée sur la scalimétrie et appliquée à ces mêmes truites sauvages de la Vèbre donne:

$\mathrm{L}_{\infty}=41,5$ et $\mathrm{k}=0,191$

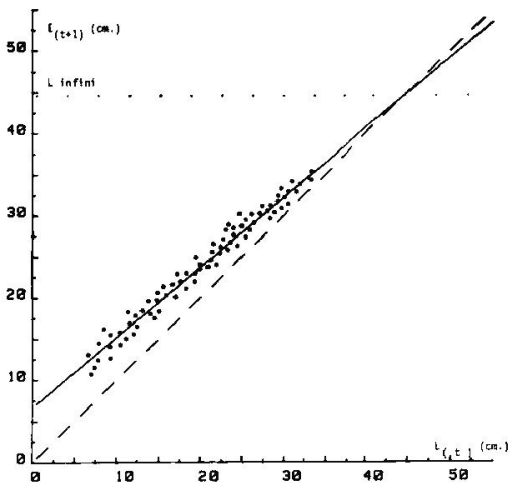

Fig. 8 : Evaluations de $\mathrm{K}$ et $\mathrm{L}$ utilisant les croissances annuelles de poisssons marqués : truites sauvages de la Vèbre.

Les valeurs obtenues à la suite des marquages étant proches, et à l'intérieur du domaine de confiance des estimations de $\mathrm{L}^{\mathrm{w}} \mathrm{et} \mathrm{k}$, on peut conclure que l'étude scalimétrique donne, de la croissance linéaire, une image concordante avec celle obtenue par les marquages.

\section{Discussion}

\subsection{Les différences de croissance entre truites sau- vages et introduites}

Le fait que la croissance des sujets introduits soit plus rapide que celle des sujets autochtones peut s'expliquer de plusieurs façons. Comme déjà dit, les poissons d'élevage mesurent 4 à $5 \mathrm{~cm}$, lorsqu'ils sont déversés en Avril et Mai, au lieu de 2 à $3 \mathrm{~cm}$ chez les sujets sauvages de même âge. Ceci est dû à un élevage dans une pisciculture alimenté par une eau de source de température constante et plus chaude $\left(11^{\circ} \mathrm{C}\right)$ que celle des rivières considérées. Il en résulte que les poissons d'élevage sont plus résistants à la prédation et aux crues que les sujets sauvages. En effet, durant la présente étude, il a été possible d'observer leur comportement lors des déversements : dès qu'ils sont mis à l'eau, ils vont se 
cacher en quelques secondes sous les galets les plus proches. Ensuite, ils développent très vite un comportement actif de chasse et capturent des proies dans des courants de bordure relativement rapides. Ils disparaissent immédiatement sous les galets lorsque l'observateur se fait repérer. Cette observation est d'autant plus nette que le courant est lent comme dans la Vèbre.

La différence de taille moyenne entre les individus introduits et les individus sauvages augmente avec l'âge: la croissance plus rapide des truites introduites est peut être due principalement à leur comportement alimentaire. Malheureusement, une étude détaillêe de celui-ci avec examen des contenus stomacaux n'a pas été possible dans le cadre de la présente étude car il était interdit de sacrifier les poissons.

Le probleme de l'alimentation de sujets introduits a été évoqué notamment par Elliot (1967), Egglishaw (1970), Egglishaw \& Shackley $(1977$; 1982) qui insistent en outre sur les différences de régime alimentaire entre les individus vivant en eaux calmes et ceux vivant en eaux courantes peu profondes; dans ces dernières, l'abondance d'invertébrés dérivant peu recherchés par les individus sauvages mais prisés par ceux introduits, peut expliquer la croissance rapide de ces derniers une fois franchie la phase d'adaptation au milieu naturel ; ce point nécessiterait une étude complète.

\subsection{Choix du modèle de Von Bertalanffy et de la méthode de Kimura}

La méthode de Kimura (1980) permet à l'évidence d'estimer les paramètres $\mathrm{K}, \mathrm{L} \propto$ et to. Cependant, la "reparamétrisation " ainsi obtenue du modèle de Von Bertalanffy ne permet pas de lui donner un comportement quasi-linéaire (Ratkowsky 1986) ; l'estimation des paramètres et de leurs intervalles de confiances est alors légèrement biaisée.

Selon cet auteur, la paramétrisation de l'équation de Von Bertalanffy proposée par Schnute \& Fournier (1980) est actuellement la meilleure. Le modèle ainsi transformé possède selon Ratkowsky (1986) un comportement quasi linéaire engendrant peu de biais dans l'évaluation des estimateurs par la méthode des moindres carrés et permettant ainsi d'estimer les intervalles de confiance en respectant les hypothèses de normalité.
En outre, le modèle proposé par Schnute \& Fournier (1980) permet de revenir à des paramètres qui ont une réelle signification biologique (les longueurs minimales et maximales observées dans l'échantillon analysé). Toutefois le modèle de Schnute \& Fournier (1980) ne permet pas la comparaison de différentes courbes de croissance comme on peut le faire par la méthode de Kimura qui s'imposait donc dans le présent travail.

\subsection{Le problème de la comparaison des croissances}

La comparaison des croissances en se référant aux paramètres de l'équation de Von Bertalanffy impose d'examiner simultanément les valeurs des paramè. tres $\mathrm{K}$ et $\mathrm{L} \alpha$; c'est ce que visent à faire la méthode de Kimura (1980) employée ici et celle de Gallucci \& Guinn (1979). Ces deux méthodes nécessitent que les valeurs calculées de $t_{0}$ soient identiques ou au moins très proches ou non significativement différentes.

Pour s'affranchir de cette dernière contrainte, un indice de croissance calculé à partir de $\mathrm{L}$ e et $\mathrm{k}$ a été recherché par plusieurs auteurs notamment Gallucci \& Quinn (1979), Pauly (1981), Pauly \& Munro (1984). De tels indices sont destinés à évaluer un potentiel de croissance pour une espèce ou une population. Il a été montré que l'index $\mathrm{O}^{\prime}=\log _{10} \mathrm{k}$ $+2 \log _{10}$ L (Pauly \& Munro, 1984) était le mieux adapté pour cela (Moreau \& al., 1986). Il serait donc judicieux de l'utiliser pour l'étude des stocks de truites à partir desquels sont opérés les repeuplements réguliers des rivières surexploitées par les pêches de loisir ; il sera ainsi possible de choisir les stocks à plus forte croissance potentielle pour l'aménagement de ces cours d'eau très recherchés par les pêcheurs sportifs.

\section{Conclusion}

A la faveur de notre étude des populations de truites, des différences de croissance entre les sujets sauvages et les poissons issus de pisciculture ont pu être mises en évidence ; la croissance se révèle plus rapide chez les individus venus de pisciculture. L'emploi de la méthode de Kimura permet de mettre en évidence de telles différences pour lesquelles il faut rechercher, au moins en partie une origine 
alimentaire comme cela a été mentionné par plusieurs auteurs cités plus haut. Par ailleurs, en géné. ralisant ce type d'observations à d'autres milieux où cohabitent, de façon durable, des sujets de piscicul. ture et des individus sauvages, on sera progressivement en mesure de comprendre l'impact des déversements de poissons sur les populations préexistantes.

Enfin, il faudra pouvoir choisir les souches de truites qui servent à l'empoissonnement des parcours de pêche en se basant notamment sur les potentialités de croissance.

\section{Remerciements}

Les auteurs remercient Messieurs Viguier, Menadié et Lassali, gardes-péche et leurs équipes pour leur aide précieuse sur le terrain.

\section{Travaux cttés}

Abad (N.). 1982. - Ecologie ef dynamique des populations de truites communes (S. trutta fario) du bassin du Tam. Thèse Doc. $3^{e}$ cycle I.N.P. Toulouse : $212 \mathrm{p}$.

Beall (E.). 1979. - Analyse scalimétrique d'une population de truites de mer (Salmo trutta L.). Iles Kerguelen, T.A.A.F. These de 3 eycle, U.P.S. Toulouse : $168 \mathrm{p}$

Campbell (R.N.). 1971. - The brown trout Salmo trutta (L) in northern Scottish lochs with special reference to the improvement of fisheries. J. Fish. Biol., 3: 1-28.

Daget (J.) \& Le Guen (J.C.). 1975. - Les critères d'àge chez les poissons p. 253-292. in Lamotte (M.) et Bourlière (F.). La démographie des populations de vertebres, Masson, Paris, 253-292.

Egglishaw (H.J.). 1970. - Production of salmon and trout in a stream in Scotland. J. Fish Biol. 2:117-136.

Egglishaw (H.J.) \& Shackley (P.E.). 1977. - Growth survival and production of juvenile Salmo and trout in a Scottish stream 1966-1975. J. Fish Biol 11:647-672.

Egglishaw (H.J.) \& Shackley (P.E.). 1982. - Influence of water depth on dispersion of juvenile Salmonid, J. Salar and $S$. trutta in a Scottish stream. J. Fish. Biol. $21: 141-155$.

Elliott (J.M.). 1067 . Invertebrate drift in a Dortmoor stream. Arch Hydrotuon, ;3:20<-237.
FAO/EIFAC/CECPI, 1984 - Documents présentés au Symposium sur l'Amélioration des stocks dans le cadre des pêcheries en eaux douces. Budapest 31 Mai-2 Juin 1982. FAO/EIFAC Tech. Pap. 42, Suppl. Vol : 554 pp.

Gallucci (V.) \& Ouinn (T.J.). 1979. - Reparameterizing. Fitting and Testing a Simple growth model. Trans. Amer. Fish. Soc., 108 : 1425 .

Grover (J.H.). (Ed.). 1980. - Allocation of Fisheries Resources. Publ. FAO/AFS. Auburn University Alabama. U.S.A. : 624 pp.

Jones (R.). 1979. - Materials and methods used in marking experiments in fisheries research. FAO Fish. Tech. Pap. (190): $136 \mathrm{pp}$.

Kimura (D.K.). 1980, - Likelyhood methods for the von Bertalanffy growth curve. Fish. Bull. U.S.A., 77.(4) : 765-776.

Lamarque (P.), Therezien (Y.) \& Charlon (N.). 1975. - Etude des conditions de la pêche electrique dans les eaux tropicales. Bull. Cent. Eud. Rech. Sci. Biarritz, 10 (3) ; $403-554$.

Moreau (J.). 1987, - Mathematical Expression of growth in Fishes recent trends and further developments pp. 81.113. In R.C. Summerfelt and G.C. Hall. Lowa Editors * Age and growth of Fishes "University, Press. Amer, U.S.A.

Moreau (J.), Bambino (C.) \& Pauly (D.). 1986. - Toward a compa. raison of 4 indices of growth performances. Reference to 100 tilapias populations. Proc. 1st Fonum of Astan Fish. Soc. May 1986 Manila The Philippines : $12 \mathrm{p}$.

Munro (J.) \& Pauly (D.). 1983. - A simple method for comparing the growth of fishes and invertebrates. Fishbyte 1 (1) : 5-6.

Nisbet (M.) \& Verneaux (J.). 1970. - Composantes chimiques des eaux courantes. Discussion et propositions de classes en tant que base d'interpretation des analyses chimiques. An $/ \mathrm{ls}$. Limpol., 6 (2) : $161-190$.

Pauty (D.). 1981. - The relationship between gill surface area and growth performance in fish : a generalization of Von Bertalanffy's theory of growth. Meerforsch. 28: 251-282.

Pauly (D.) \& Munro (J.). 1984. - Once more on growth comparisons in fishes and inverte-brates. Fishbvte, 2 (1); 21-22.

Ratkowski (J.). 1986. - Statistical Properties of Altemative Parameterization of the von Bertalanffy Growth Curve : Can, J. Fish. Aquat. Sci., $43: 742-747$.

Schnute (J.) \& Fournier (D.). 1980. - A new approach to length frequency analysis : growth structure. Can. J. Fish. Aquat. Sci., 37: $1337-1351$.

Thibault (M.). 1983. - Les transplantations de Salmonidés d'eau courante en France, Saumon Atlantique (Salmo Salar L.) et truite commune (Salmo trutta L) C.R. Soc. Biogéogr, 59 (3c) : $405-420$.

Vibert (R.). 1968. - Application de l'électricité à la biologie et à l'aménagement des pêches continentales. Acte Symp. FAO, Bel. grade, Ed. INRA. Paris : 276 p. 